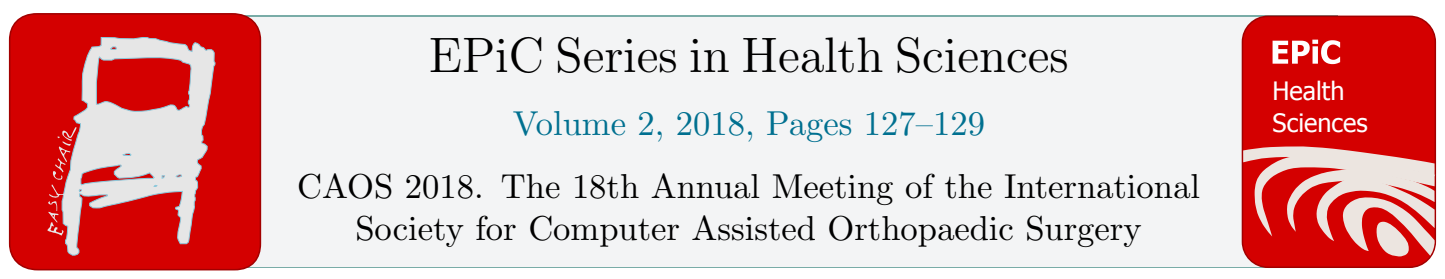

\title{
Method of auto-calibration and auto- registration of an intra-operative 3D imaging system integrated with navigation
}

\author{
Stéphane Lavallée ${ }^{1,}$ Laurence Van Beek ${ }^{1}$, David Armand ${ }^{1}$, Arnaud Pierre ${ }^{1}$, \\ John Sledge ${ }^{2}$, Lou Jenis ${ }^{3}$, Jérôme Tonetti ${ }^{4}$ \\ ${ }^{1}$ Cimagine Medical, Grenoble FR \\ ${ }^{2}$ Lafayette Bone \& Joint Clinic, Lafayette LA \\ ${ }^{3}$ Newton-Wellesley Hospital, Newton MA \\ ${ }^{4}$ Grenoble University Hospital FR \\ sl@stephanelavallee.com, laurence.vanbeek@surgivisio.com, \\ david.armand@surgivisio.com, david.armand@surgivisio.com, isledge@lbjclinic.com, \\ ljenis@partners.org , jtonetti@chu-grenoble.fr
}

\begin{abstract}
This paper describes novel methods of auto-calibration and auto-registration of 3D imaging and navigation, implemented in a fully integrated device made of a motorized $\mathrm{C}$-arm and a navigation system. Preliminary results are presented. Benefits in terms of saved time, increased accuracy, reasonable x-ray dose, and surgeon satisfaction are reported.
\end{abstract}

\section{Introduction}

Intra-operative 3D imaging has been widely used clinically for spine surgery in conjunction with surgical navigation for more than a decade. Clinical benefits have been demonstrated by significant reduction of misplaced pedicular screws in both open surgery or MIS [1,2]. However, several pitfalls have been reported in the literature [3] when two distinct devices are used, one device for capturing a 3D intra-operative image of the patient, a second device for navigation or robotics. Generally, pitfalls relate to patient tracker motion, inaccuracies, additional time, difficulties of use for non-experienced staff, etc. As a result, in most clinical centers, existing devices coupling 3D imaging and navigation are not used for all spine cases, but only for some specific cases, and the selection of such cases depends on the surgical team experience, support, habits, and case difficulty. To overcome these difficulties, authors have proposed to develop a novel system, which is a fully integrated device that merges a $2 \mathrm{D}$ imaging $\mathrm{C}$-arm with 5 motorized axes, 3D imaging reconstruction and navigation, with one user 
interface to handle the complete system on a tablet and one core computing system to handle all functions of the device (x-rays, image detection, C-arm motorization, user interface, tracking and navigation, etc.). Integrated instruments are further used to enable auto-calibration and auto-registration methods as described below.

\section{Methods}

The methods presented in this paper have been implemented on the Surgivisio 3D C-arm/Navigation device (Surgivisio, Cimagine Medical, France).

During an open surgery or percutaneous procedure, a patient reference is fixed to the spine, generally on the spinous processes, using small sharp pins that are linked together rigidly by a constrained fixation mechanism. A single-use calibration phantom is placed on the patient reference. A 2D frontal image is acquired. From this single 2D image, the calibration phantom is detected automatically and an estimate of the region of interest is determined accordingly in 3D. An optimized C-arm trajectory is then computed automatically and launched to maximize the reconstruction volume, avoid collisions of the $\mathrm{C}$-arm with the OR table and reduce the $\mathrm{x}$-ray dose. To further reduce $\mathrm{x}$-ray dose to a reasonably acceptable level for the patient, only 90 or 180 projections are acquired. Note that the surgical staff goes outside of the room during image acquisition and therefore is not subject to x-ray radiation. The calibration phantom is detected automatically on all projections, even in the presence of missing fiducials. The set of projections is automatically calibrated intrinsically and extrinsically with respect to the calibration phantom. Using this auto-calibration method, the 3D image is reconstructed by compensating any patient motion, breathing or any deformation of the $\mathrm{C}$-arm. Then, the calibration phantom is replaced by a patient optical tracker at a precisely known location, using a magnetic reproducible fixation on the patient reference. This trivial step suffices to perform auto-registration of the patient tracker with the 3D image. The surgeon uses a factory-calibrated single use trocar equipped with a tracker and just performs a system-confidence check. Finally, the surgeon can immediately visualize the trocar position on reconstructed slices in the 3D image and navigate. Once the trocar has been inserted in a pedicle, the standard surgical procedure continues. Fig 1 illustrates the global process.

\section{Results}

This paper presents only preliminary pre-clinical and clinical results.

The Surgivisio device implementing the methods presented in this paper has been used successfully on more than 53 procedures on specimens during pre-clinical validation and 14 clinical cases after $\mathrm{CE}$ marking was obtained. The methods of auto-calibration and auto-registration described in this paper were used successfully in all cases.

Users expressed a high degree of confidence and satisfaction, from the very first clinical cases.

The accuracy of the system has been reported in [4]. Testing on phantoms, with induced phantom motion during $3 \mathrm{D}$ image acquisition to simulate breathing, shows that the complete accuracy of the navigated trocar is in the range of the voxel size $(0.35 \mathrm{~mm})$.

Once the system is set-up, the procedure time from the first 2D image to surgical instrument navigation is less than 5 minutes. This is a major benefit of system integration.

In specimen study, the average $\mathrm{x}$-ray dose-area product (DAP) per 90/180 images 3D reconstruction was $1810 \pm 750 / 3180 \pm 1430 \mathrm{mGy} . \mathrm{cm}^{2}$ for lumbar spine, $1740 \pm 970 / 3580 \pm 2320 \mathrm{mGy} . \mathrm{cm}^{2}$ for thoracic spine, $1760 \pm 900 / 2530 \pm 680 \mathrm{mGy} . \mathrm{cm}^{2}$ for sacrum. First clinical results present an average DAP per 3D acquisition comprised between 3000 and $6530 \mathrm{mGy} \cdot \mathrm{cm}^{2}$. 


\section{Discussion}

This paper has described novel and patented methods of auto-calibration and auto-registration of 3D imaging and navigation, and their benefits in terms of saved time, increased accuracy, reasonable $\mathrm{x}$-ray dose, and surgeon satisfaction. Clinical studies will be necessary to confirm these preliminary excellent results.

There is no doubt that these benefits are the direct result of full system integration. At the contrary, coupling of devices offers modularity benefits at the expense of multiple user interfaces, complex user actions to interface devices together, multiple and complex support and training, and suboptimal methods, which may generate surgeon and staff frustration. This could be an explanation for the lack of $100 \%$ clinical routine use of these technologies at the present time.

\section{References}

[1] Grützner, P. A. et al. Intraoperative three-dimensional navigation for pedicle screw placement. Chirurg 75, 967-975 (2004).

[2] Silbermann, J. et al. Computer tomography assessment of pedicle screw placement in lumbar and sacral spine: comparison between free-hand and O-arm based navigation techniques. Eur. Spine J. 20, 875-81 (2011).

[3] G. Rahmathulla, E. W. Nottmeier, S. M. Pirris, H. G. Deen, M. A. Pichelmann, Intraoperative imageguided spinal navigation: technical pitfalls and their avoidance, Neurosurg Focus 36 (3):E3. (2014).

[4] J. B. Stiehl, L. Van Beek, F. Bonetti, S. Lavallee, D. Armand, A. Pierre, C. Jouvie; Precision of Motion Limiting Software Algorithm in Imaged Guided Automated Navigation, ORS 2016 Annual Meeting Poster No. 1689 (2016)

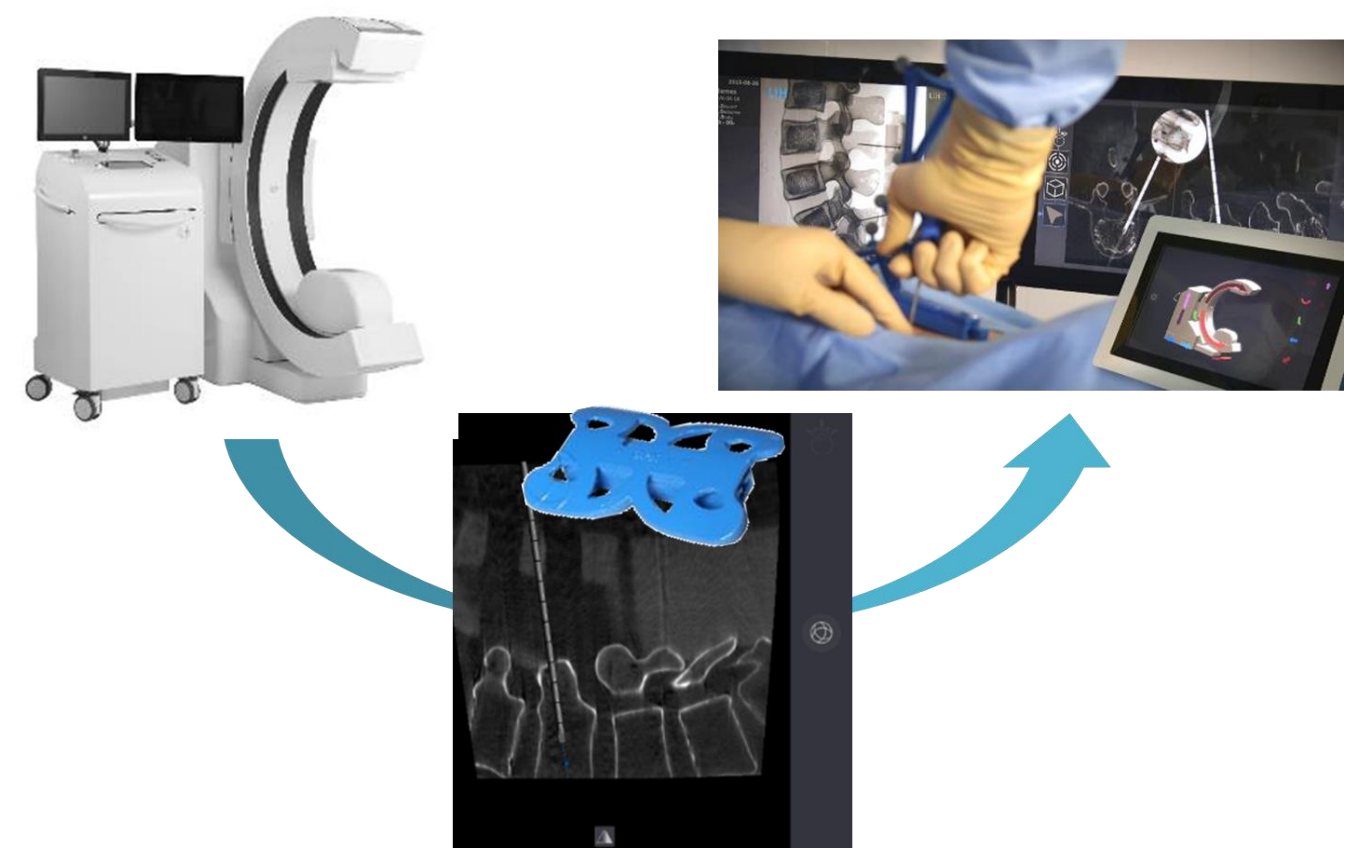

Figure 1. Illustration of the auto-calibration and auto-registration method implemented on the Surgivisio system. 